\title{
THREE-DIMENSIONAL FLUCTUATING COUETTE FLOW THROUGH THE POROUS PLATES WITH HEAT TRANSFER
}

\author{
M. GURIA AND R. N. JANA
}

Received 23 March 2006; Accepted 23 March 2006

Unsteady Couette flow of a viscous incompressible fluid between two horizontal porous flat plates is considered. The stationary plate is subjected to a periodic suction and the plate in uniform motion is subjected to uniform injection. Approximate solutions have been obtained for the velocity and the temperature fields, skin friction by using perturbation technique. The heat transfer characteristic has also been studied on taking viscous dissipation into account. It is found that the main flow velocity decreases with increase in frequency parameter. On the other hand, the magnitude of the cross-flow velocity increases with increase in frequency parameter. It is seen that the amplitude of the shear stress due to main flow decreases while that due to cross-flow increases with increase in frequency parameter. It is also seen that the tangent of phase shifts both due to the main and cross-flows decrease with increase in frequency parameter. It is observed that the temperature increases with increase in frequency parameter.

Copyright (c) 2006 Hindawi Publishing Corporation. All rights reserved.

\section{Introduction}

Couette flow is important in numerous mechanisms involving the relative motion of two surfaces. The problem of Couette flow is considered important in transpiration cooling. In this process several engines can be protected from the influence of hot gases. This process is used in turbojet and rocket engines, like combustion chamber walls, exhaust nozzles, and gas turbine blades. The solution is well known when both surfaces are flat and moving in their own planes. An exact solution of Navier-Stokes equations between two parallel plates without suction is well known in Schlichting [3]. Two-dimensional plane Couette flow with transpiration cooling applying uniform injection and suction at the porous plates is discussed by Eckert [1]. Gersten and Gross [2] studied the threedimensional flow and heat transfer along a flat plate by applying periodic suction. Singh et al. [7] analyzed the three-dimensional flow and heat transfer past a vertical wall. Singh et al. [6] and Singh [4] studied the three-dimensional flow and heat transfer past a porous 
plate. Recently Singh [5] discussed the Couette flow between two parallel plates with transverse sinusoidal injection of the fluid at the stationary plate and the constant suction at the plate in motion.

However, the application of the transverse sinusoidal injection or suction velocity in the unsteady problem of transpiration cooling has not yet received much attention. In the present paper we have studied the unsteady Couette flow and heat transfer between two horizontal parallel porous flat plates with periodic suction at the stationary plate and constant injection at the plate in motion. We assume that the periodic suction velocity is time-dependent and perpendicular to the flow direction. Due to the periodic suction the flow becomes three dimensional. The main flow velocity profile, cross-flow velocity profile, and shear stress have been calculated and plotted. The heat transfer characteristic has also been studied on taking viscous dissipation into account. It is found that the main flow velocity increases with the increase in either Reynolds number or suction parameter, while it decreases with the increase in frequency parameter. On the other hand, the magnitude of the cross-flow velocity increases with the increase in either suction parameter or frequency parameter, while it increases near the stationary plate and decreases near the moving plate with the increase in Reynolds number. It is seen that the amplitude of the shear stress due to main flow increases with the increase in Reynolds number but decreases with the increase in frequency parameter. The phase shift decreases with the increase in frequency parameter. But for very small values of Reynolds number, it increases and decreases for large Reynolds number. Also it is seen that the amplitude of the shear stress due to cross-flow increases with the increase in either Reynolds number or frequency parameter. The phase shift decreases with the increase in frequency parameter while it increases with the increase in Reynolds number. It is observed that the temperature increases with the increase in frequency parameter, on the other hand, it increases near the stationary plate and decreases near the moving plate with the increase in Reynolds number.

\section{Formulation of the problem}

Consider the unsteady flow of a viscous incompressible fluid between two horizontal flat porous plates separated by a distance $d$. The upper plate moves with a uniform velocity $U$ in the direction of the flow. We choose a cartesian coordinate system with its origin on the lower stationary plate, $x^{\star}$-axis is in the direction of the flow, $y^{\star}$-axis is perpendicular to the plate, and $z^{\star}$-axis normal to the $x^{\star} y^{\star}$-plane.

The upper plate is subjected to a constant injection $-V_{0}$ and the lower plate to a transverse sinusoidal suction velocity distribution of the form

$$
v^{\star}=-V_{0}\left[1+\epsilon \cos \left(\frac{\pi z^{\star}}{d}-c t^{\star}\right)\right]
$$

where $\epsilon(\ll 1)$ is the amplitude of the suction velocity. Denoting velocity components $u^{\star}, v^{\star}, w^{\star}$ in the directions $x^{\star}-, y^{\star}$-, and $z^{\star}$-axes, respectively, the flow is governed by 
the following equations:

$$
\begin{gathered}
\frac{\partial v^{\star}}{\partial y^{\star}}+\frac{\partial w^{\star}}{\partial z^{\star}}=0, \\
\frac{\partial u^{\star}}{\partial t^{\star}}+v^{\star} \frac{\partial u^{\star}}{\partial y^{\star}}+w^{\star} \frac{\partial u^{\star}}{\partial z^{\star}}=v\left(\frac{\partial^{2} u^{\star}}{\partial y^{\star 2}}+\frac{\partial^{2} u^{\star}}{\partial z^{\star 2}}\right), \\
\frac{\partial v^{\star}}{\partial t^{\star}}+v^{\star} \frac{\partial v^{\star}}{\partial y^{\star}}+w^{\star} \frac{\partial v^{\star}}{\partial z^{\star}}=-\frac{1}{\rho} \frac{\partial p^{\star}}{\partial y^{\star}}+v\left(\frac{\partial^{2} v^{\star}}{\partial y^{\star 2}}+\frac{\partial^{2} v^{\star}}{\partial z^{\star 2}}\right), \\
\frac{\partial w^{\star}}{\partial t^{\star}}+v^{\star} \frac{\partial w^{\star}}{\partial y^{\star}}+w^{\star} \frac{\partial w^{\star}}{\partial z^{\star}}=-\frac{1}{\rho} \frac{\partial p^{\star}}{\partial z^{\star}}+v\left(\frac{\partial^{2} w^{\star}}{\partial y^{\star 2}}+\frac{\partial^{2} w^{\star}}{\partial z^{\star 2}}\right),
\end{gathered}
$$

where $v$ is the kinematic viscosity, $\rho$ is the density, $p^{\star}$ is the fluid pressure.

The boundary conditions of the problem are

$$
\begin{gathered}
u^{\star}=0, \quad v^{\star}=-V_{0}\left[1+\cos \left(\frac{\pi}{d} z^{\star}-c t^{\star}\right)\right], \quad w^{\star}=0 \quad \text { at } y^{\star}=0, \\
u^{\star}=U, \quad v^{\star}=-V_{0}, \quad w^{\star}=0 \quad \text { at } y^{\star}=d .
\end{gathered}
$$

Introducing the nondimensional variables

$$
y=\frac{y^{\star}}{d}, \quad z=\frac{z^{\star}}{d}, \quad t=c t^{\star}, \quad p=\frac{p^{\star}}{\rho U^{2}}, \quad u=\frac{u^{\star}}{U}, \quad v=\frac{v^{\star}}{U}, \quad w=\frac{w^{\star}}{U},
$$

equation (2.2) becomes

$$
\begin{gathered}
\frac{\partial v}{\partial y}+\frac{\partial w}{\partial z}=0 \\
\omega \frac{\partial u}{\partial t}+\operatorname{Re}\left(v \frac{\partial u}{\partial y}+w \frac{\partial u}{\partial z}\right)=\frac{\partial^{2} u}{\partial y^{2}}+\frac{\partial^{2} u}{\partial z^{2}} \\
\omega \frac{\partial v}{\partial t}+\operatorname{Re}\left(v \frac{\partial v}{\partial y}+w \frac{\partial v}{\partial z}\right)=-\operatorname{Re} \frac{\partial p}{\partial y}+\left(\frac{\partial^{2} v}{\partial y^{2}}+\frac{\partial^{2} v}{\partial z^{2}}\right) \\
\omega \frac{\partial w}{\partial t}+\operatorname{Re}\left(v \frac{\partial w}{\partial y}+w \frac{\partial w}{\partial z}\right)=-\operatorname{Re} \frac{\partial p}{\partial z}+\left(\frac{\partial^{2} w}{\partial y^{2}}+\frac{\partial^{2} w}{\partial z^{2}}\right)
\end{gathered}
$$

where $\operatorname{Re}=U d / \nu$, the Reynolds number; $S=V_{0} / U$, the suction parameter; and $\omega=$ $c d^{2} / \nu$, the frequency parameter. Using (2.4) the boundary conditions (2.3) become

$$
\begin{gathered}
u=0, \quad v=-S[1+\epsilon \cos (\pi z-t)], \quad w=0 \quad \text { at } y=0, \\
u=1, \quad v=-S, \quad w=0 \quad \text { at } y=1 .
\end{gathered}
$$


4 Three-dimensional Couette flow with heat transfer

\section{Solution of the problem}

In order to solve the differential equation (2.5), we assume the solution of the following form:

$$
\begin{gathered}
u=u_{0}(y)+\epsilon u_{1}(y, z, t)+\epsilon^{2} u_{2}(y, z, t)+\cdots, \\
v=v_{0}(y)+\epsilon v_{1}(y, z, t)+\epsilon^{2} v_{2}(y, z, t)+\cdots, \\
w=w_{0}(y)+\epsilon w_{1}(y, z, t)+\epsilon^{2} w_{2}(y, z, t)+\cdots, \\
p=p_{0}(y)+\epsilon p_{1}(y, z, t)+\epsilon^{2} p_{2}(y, z, t)+\cdots .
\end{gathered}
$$

When $\epsilon=0$ the flow becomes two-dimensional with constant suction and injection at both the plates. In this case (2.5) is reduced to

$$
\begin{gathered}
v_{0}^{\prime}=0, \\
u_{0}^{\prime \prime}+S \operatorname{Re} u_{0}^{\prime}=0,
\end{gathered}
$$

where the primes denote differentiation with respect to $y$, and the corresponding boundary conditions are

$$
\begin{array}{ll}
u_{0}=0, & v_{0}=-S \quad \text { at } y=0, \\
u_{0}=1, & v_{0}=-S \quad \text { at } y=1 .
\end{array}
$$

The solutions of (3.2) using (3.3) become

$$
v_{0}(y)=-S, \quad u_{0}(y)=\frac{1-e^{-S \operatorname{Re} y}}{1-e^{-S \operatorname{Re}}}
$$

When $\epsilon \neq 0$, for small values of $\epsilon$, we take only upto $O(\epsilon)$. Substituting (3.1) in (2.5) and comparing the coefficients of $\epsilon$ from both sides, we get

$$
\begin{gathered}
\frac{\partial v_{1}}{\partial y}+\frac{\partial w_{1}}{\partial z}=0 \\
\omega \frac{\partial u_{1}}{\partial t}+\operatorname{Re}\left(-S \frac{\partial u_{1}}{\partial y}+v_{1} \frac{\partial u_{0}}{\partial y}\right)=\frac{\partial^{2} u_{1}}{\partial y^{2}}+\frac{\partial^{2} u_{1}}{\partial z^{2}} \\
\omega \frac{\partial v_{1}}{\partial t}-S \operatorname{Re} \frac{\partial v_{1}}{\partial y}=-\operatorname{Re} \frac{\partial p_{1}}{\partial y}+\frac{\partial^{2} v_{1}}{\partial y^{2}}+\frac{\partial^{2} v_{1}}{\partial z^{2}} \\
\omega \frac{\partial w_{1}}{\partial t}-S \operatorname{Re} \frac{\partial w_{1}}{\partial y}=-\operatorname{Re} \frac{\partial p_{1}}{\partial z}+\frac{\partial^{2} w_{1}}{\partial y^{2}}+\frac{\partial^{2} w_{1}}{\partial z^{2}}
\end{gathered}
$$


The corresponding boundary conditions are reduced to

$$
\begin{gathered}
u_{1}=0, \quad v_{1}=-S \cos (\pi z-t), \quad w_{1}=0 \quad \text { at } y=0, \\
u_{1}=0, \quad v_{1}=0, \quad w_{1}=0 \quad \text { at } y=1 .
\end{gathered}
$$

These are the linear partial differential equations describing the three-dimensional flow. To solve (3.6), (3.7), and (3.8) we assume $u_{1}, v_{1}, w_{1}$, and $p_{1}$ of the following form:

$$
\begin{aligned}
u_{1}(y, z, t) & =u_{11}(y) e^{i(\pi z-t)}, \\
v_{1}(y, z, t) & =v_{11}(y) e^{i(\pi z-t)}, \\
w_{1}(y, z, t) & =\frac{i}{\pi} v_{11}^{\prime}(y) e^{i(\pi z-t)}, \\
p_{1}(y, z, t) & =p_{11}(y) e^{i(\pi z-t)} .
\end{aligned}
$$

The corresponding boundary conditions are

$$
\begin{gathered}
u_{11}=0, \quad v_{11}=-S, \quad v_{11}^{\prime}=0 \quad \text { at } y=0, \\
u_{11}=0, \quad v_{11}=0, \quad v_{11}^{\prime}=0 \quad \text { at } y=1 .
\end{gathered}
$$

Substituting (3.10) in (3.6), (3.7), and (3.8), we get the following differential equations:

$$
\begin{gathered}
u_{11}^{\prime \prime}+S \operatorname{Re} u_{11}^{\prime}-\left(\pi^{2}-i \omega\right) u_{11}=\operatorname{Re} v_{11} u_{0}^{\prime}, \\
v_{11}^{\prime \prime}+S \operatorname{Re} v_{11}^{\prime}-\left(\pi^{2}-i \omega\right) v_{11}=\operatorname{Re} p_{11}^{\prime}, \\
v_{11}^{\prime \prime \prime}+S \operatorname{Re} v_{11}^{\prime \prime}-\left(\pi^{2}-i \omega\right) v_{11}^{\prime}=\operatorname{Re} \pi^{2} p_{11} .
\end{gathered}
$$

Solving (3.12) under the boundary conditions (3.11), we get

$$
\begin{gathered}
u_{1}(y, z, t)=\left[A_{3} e^{-r_{1} y}+A_{4} e^{-r_{2} y}+C_{1} e^{-\left(r_{1}+S \mathrm{Re}\right) y}+C_{2} e^{-\left(r_{2}+S \mathrm{Re}\right) y}\right. \\
\left.+C_{3} e^{(\pi-S \mathrm{Re}) y}+C_{4} e^{-(\pi+S \mathrm{Re}) y}\right] e^{i(\pi z-t)}, \\
v_{1}(y, z, t)=\left[A e^{-r_{1} y}+B e^{-r_{2} y}+C e^{\pi y}+D e^{-\pi y}\right] e^{i(\pi z-t)}, \\
w_{1}(y, z, t)=-\frac{i}{\pi}\left[A r_{1} e^{-r_{1} y}+B r_{2} e^{-r_{2} y}-C \pi e^{\pi y}+D \pi e^{-\pi y}\right] e^{i(\pi z-t)}, \\
p_{1}(y, z, t)=\left[A_{1} e^{\pi y}+A_{2} e^{-\pi y}\right] e^{i(\pi z-t)},
\end{gathered}
$$


where

$$
\begin{gathered}
r_{1}=\frac{S \operatorname{Re}+\sqrt{S^{2} \operatorname{Re}^{2}+4\left(\pi^{2}-i \omega\right)}}{2}, \quad r_{2}=\frac{S \operatorname{Re}-\sqrt{S^{2} \operatorname{Re}^{2}+4\left(\pi^{2}-i \omega\right)}}{2}, \\
A=\frac{S e^{\pi}\left(\pi r_{4}+r_{6}\right)+S e^{-\pi}\left(r_{6}-\pi r_{4}\right)}{2\left(r_{3} r_{6}-r_{4} r_{5}\right)}, \quad B=\frac{-S e^{\pi}\left(r_{5}+\pi r_{3}\right)-S e^{-\pi}\left(r_{5}-\pi r_{3}\right)}{2\left(r_{3} r_{6}-r_{4} r_{5}\right)}, \\
C=-\frac{1}{2 \pi}\left[A\left(\pi-r_{1}\right)+B\left(\pi-r_{2}\right)+S \pi\right], \\
r_{3}=e^{-r_{1}}-\frac{\left(\pi-r_{1}\right)}{2 \pi} e^{\pi}-\frac{\left(\pi+r_{1}\right)}{2 \pi} e^{-\pi},
\end{gathered}
$$

\section{Result and discussion}

We have presented the nondimensional main flow velocity $u$ against $y$ for different values of Reynolds number Re, suction parameter $S$, and frequency parameter $\omega$ for $z=0.0$, $t=0.2, \epsilon=0.2$ in Figures 4.1, 4.2, and 4.3. From the figure it is seen that the main flow velocity $u$ increases with increase in either $\operatorname{Re}$ or $S$, while it decreases with the increase in $\omega$. The cross-flow velocity profile is shown in Figures 4.4 and 4.5 against $y$ for $z=0.5$, $t=0.2, \epsilon=0.2$. It is observed that the magnitude of the cross-flow velocity $w$ increases with the increase in either $S$ or $\omega$ but it increases near the stationary plate and decreases near the moving plate with the increase in Re. This is due to the fact that suction at the stationary plate and injection at the moving plate are two exactly opposite processes. Also 


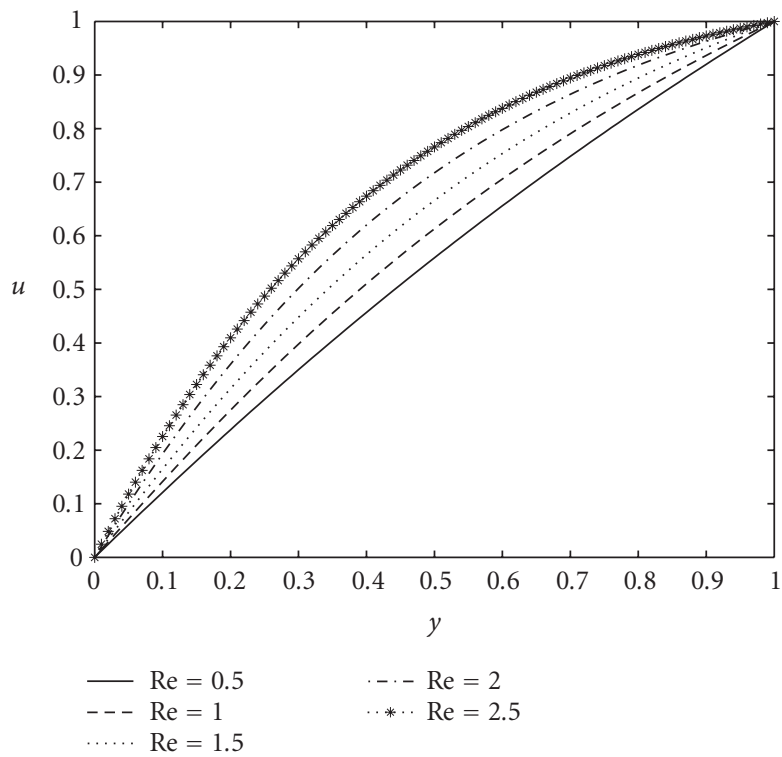

Figure 4.1. Main velocity $u$ for $\omega=6.0, S=1.0, t=0.2, z=0.0, \epsilon=0.2$.

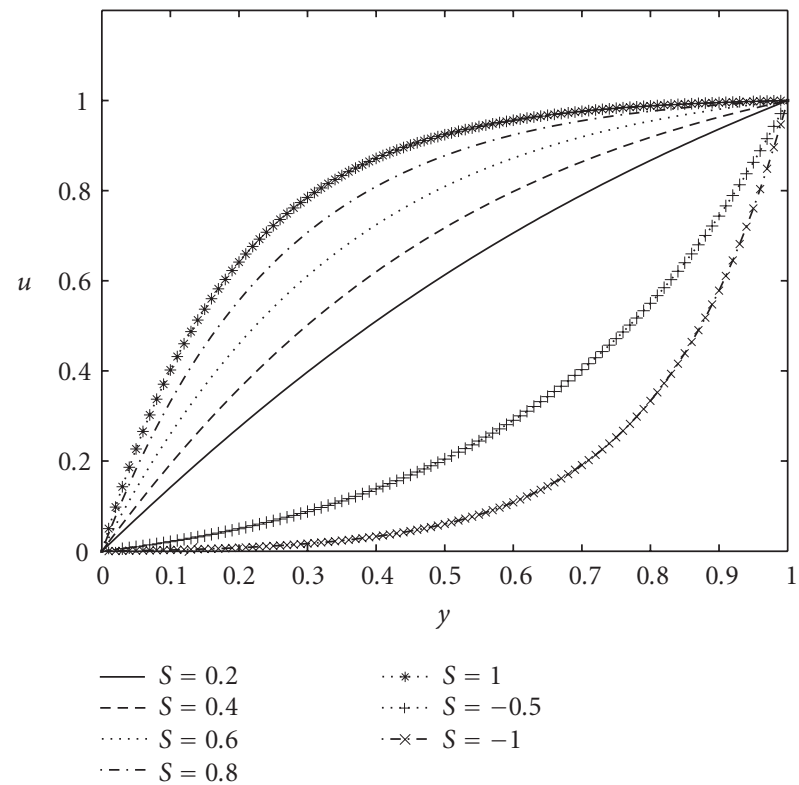

Figure 4.2. Main velocity $u$ for $\omega=6.0, \operatorname{Re}=5.0, Z=0.0, t=0.2, \epsilon=0.2$. 
8 Three-dimensional Couette flow with heat transfer

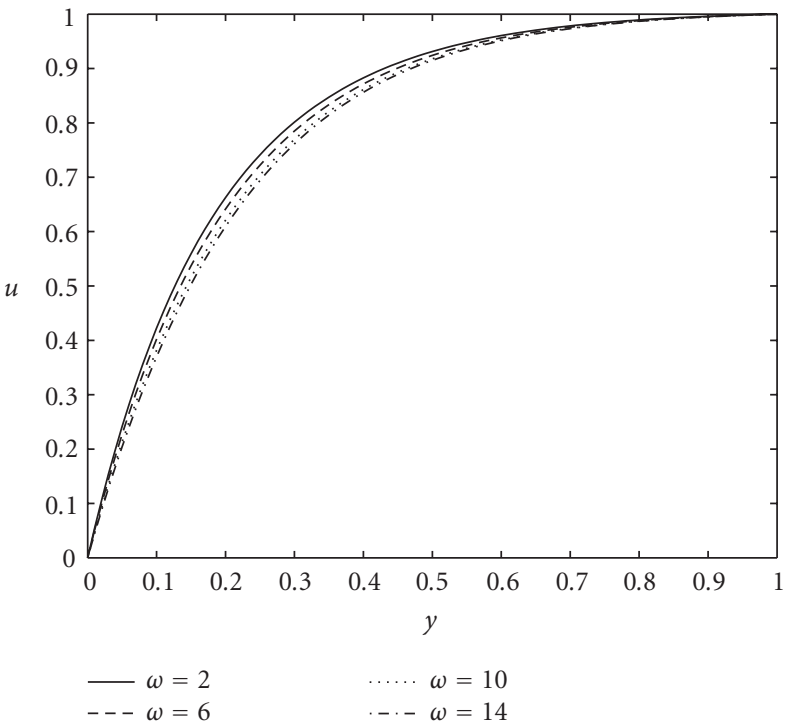

Figure 4.3. Main velocity $u$ for $S=1.0, \operatorname{Re}=5.0, t=0.2, z=0.0, \epsilon=0.2$.

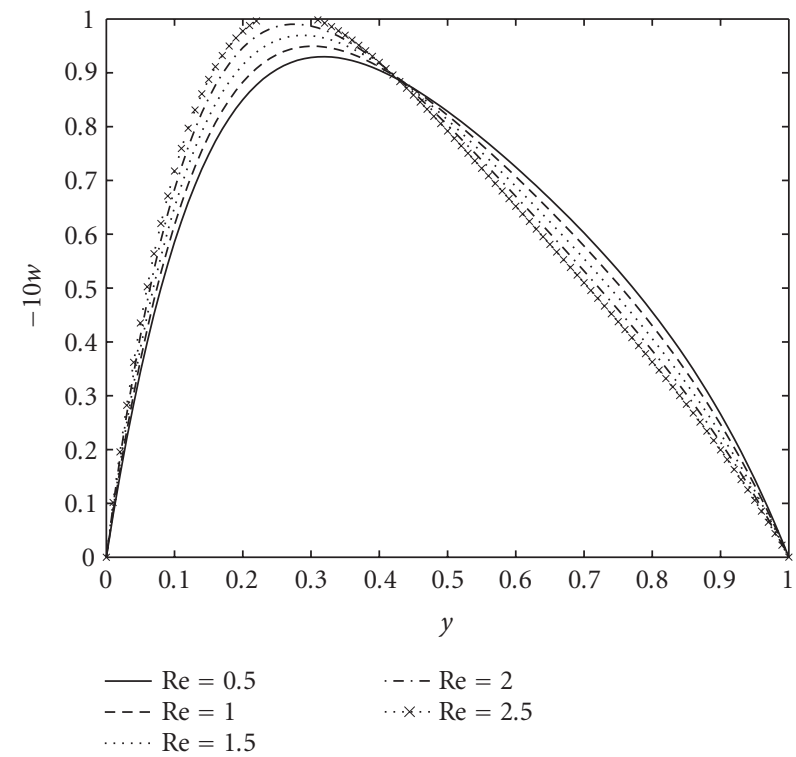

Figure 4.4. Cross-velocity $-10 w$ for $\omega=6.0, S=1.0, z=0.5, t=0.2, \epsilon=0.2$.

the variations of cross-velocity $w$ for different values of $\omega$ are shown in Table 4.1. From the table it is observed that the magnitude of $w$ increases with increase in $\omega$. 


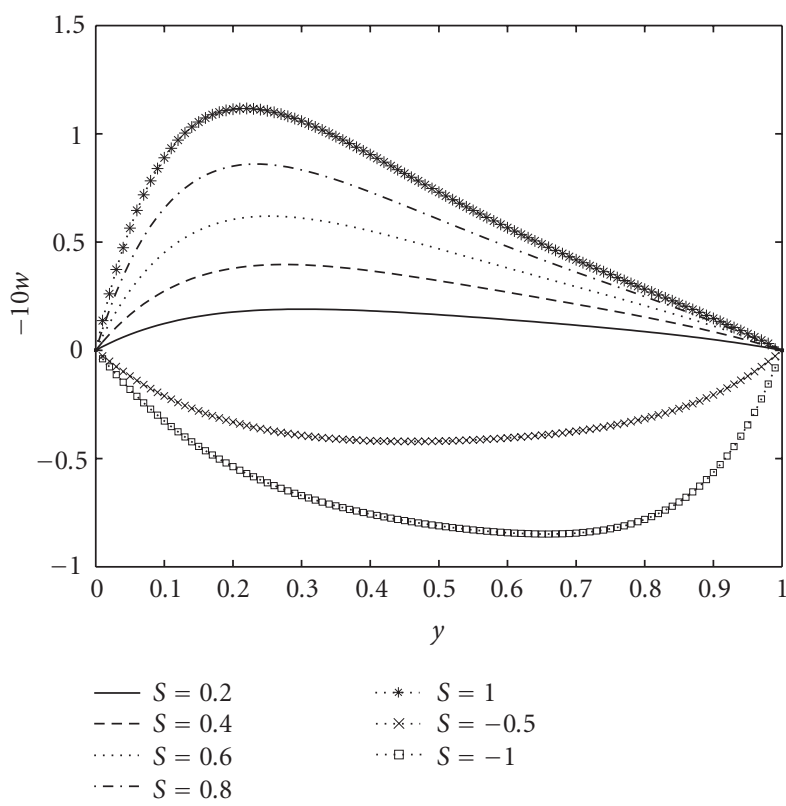

Figure 4.5. Cross-velocity $-10 w$ for $\omega=6.0, \operatorname{Re}=5.0, z=0.5, t=0.2, \epsilon=0.2$.

The nondimensional shear stress components due to the main flow and cross-flow at the plate $y=0$ are, respectively,

$$
\begin{aligned}
\tau_{x} & =\frac{d \tau_{x}^{*}}{\mu U}=\left(\frac{d u_{0}}{d y}\right)_{y=0}+\epsilon\left(\frac{d u_{1}}{d y}\right)_{y=0} \\
& =\left(\frac{d u_{0}}{d y}\right)_{y=0}+\epsilon\left(\frac{d u_{11}}{d y}\right)_{y=0} e^{i(\pi z-t)} \\
& =\tau_{u_{0}}+\epsilon \operatorname{Re}_{1} \cos \left(\pi z-t+\phi_{1}\right), \\
\tau_{z} & =\frac{d \tau_{z}^{*}}{\mu U}=\left(\frac{d w_{0}}{d y}\right)_{y=0}+\epsilon\left(\frac{d w_{1}}{d y}\right)_{y=0} \\
& =\epsilon \frac{i}{\pi}\left(\frac{d v_{11}^{\prime}}{d y}\right)_{y=0} e^{i(\pi z-t)} \\
& =\epsilon \operatorname{Re}_{2} \cos \left(\pi z-t+\phi_{2}\right),
\end{aligned}
$$

where

$$
\begin{array}{ll}
\operatorname{Re}_{1}=\left\{X_{1}^{2}+Y_{1}^{2}\right\}^{1 / 2}, & \tan \phi_{1}=\frac{Y_{1}}{X_{1}} \\
\operatorname{Re}_{2}=\left\{X_{2}^{2}+Y_{2}^{2}\right\}^{1 / 2}, & \tan \phi_{2}=\frac{Y_{2}}{X_{2}}
\end{array}
$$


10 Three-dimensional Couette flow with heat transfer

Table 4.1. Cross-flow velocity for $S=1.0, \operatorname{Re}=5.0, z=0.5, t=0.2, \epsilon=0.2$.

\begin{tabular}{ccccc}
\hline $\mathrm{y}$ & \multicolumn{3}{c}{$-10 \mathrm{w}$} \\
\hline 0.00 & $\omega=2.0$ & $\omega=6.0$ & $\omega=10.0$ & $\omega=14.0$ \\
0.20 & 0.000000 & 0.000000 & 0.00000 & 0.000000 \\
0.40 & 1.109254 & 1.113393 & 1.118991 & 1.125880 \\
0.60 & 0.907241 & 0.903539 & 0.898763 & 0.893008 \\
0.80 & 0.568972 & 0.565611 & 0.561192 & 0.555843 \\
1.00 & 0.282000 & 0.281842 & 0.281629 & 0.281395 \\
\hline
\end{tabular}

Table 4.2. Shear stress due to main flow.

\begin{tabular}{ccccccc}
\hline $\operatorname{Re}$ & \multicolumn{3}{c}{$\operatorname{Re}_{1}$} & & & $-\tan \phi_{1}$ \\
& $\omega=5.0$ & $\omega=10.0$ & $\omega=15.0$ & $\omega=5.0$ & $\omega=10.0$ & $\omega=15.0$ \\
\hline 0.4 & 0.3133 & 0.1787 & 0.1344 & 1.0171 & 1.2728 & 1.2516 \\
0.6 & 0.4892 & 0.2891 & 0.2182 & 0.6059 & 0.9704 & 1.0421 \\
0.8 & 0.6606 & 0.4119 & 0.3132 & 0.3236 & 0.7402 & 0.8681 \\
1.0 & 0.8185 & 0.5452 & 0.4191 & 0.0970 & 0.5541 & 0.7193 \\
\hline
\end{tabular}

Table 4.3. Shear stress due to cross-flow.

\begin{tabular}{ccccccc}
\hline $\operatorname{Re}$ & \multicolumn{3}{c}{$\operatorname{Re}_{2}$} & & $-\tan \phi_{2}$ & \\
\hline & $\omega=5.0$ & $\omega=10.0$ & $\omega=15.0$ & $\omega=5.0$ & $\omega=10.0$ & $\omega=15.0$ \\
\hline 0.4 & 3.9497 & 4.1925 & 4.5407 & 6.2946 & 3.3011 & 2.3627 \\
0.6 & 4.0614 & 4.2989 & 4.6407 & 6.5018 & 3.4055 & 2.4331 \\
0.8 & 4.1760 & 4.4080 & 4.7430 & 6.7209 & 3.5155 & 2.5070 \\
1.0 & 4.2937 & 4.5198 & 4.8478 & 6.9523 & 3.6316 & 2.5847 \\
\hline
\end{tabular}

The shear stresses due to main flow and cross-flow are shown in Tables 4.2 and 4.3 for $S=1.0$. From the table it is seen that the amplitude $\operatorname{Re}_{1}$ of the shear stress due to main flow increases with the increase in Re but decreases with the increase in $\omega$. The magnitude of the tangent of phase shift $\tan \phi_{1}$ decreases with the increase in $\omega$ while it increases for small values of Re and decreases for large values of Re. It is also seen from Table 4.2 that there is always a phase lag. Table 4.3 shows the shear stress due to cross-flow for $S=1.0$. It is observed that the amplitude $\mathrm{Re}_{2}$ of shear stress due to cross-flow increases with the increase in either Re or $\omega$. It is also seen that there is always a phase lag. The magnitude of the tangent of phase angle increases with the increase in Re but decreases with the increase in $\omega$. 


\section{Heat transfer}

To find the temperature distribution we consider the energy equation

$$
\frac{\partial T^{\star}}{\partial t^{\star}}+v^{\star} \frac{\partial T^{\star}}{\partial y^{\star}}+w^{\star} \frac{\partial T^{\star}}{\partial z^{\star}}=\alpha\left(\frac{\partial^{2} T^{\star}}{\partial y^{2}}+\frac{\partial^{2} T^{\star}}{\partial z^{2}}\right)+\frac{\mu}{\rho C_{p}} \Phi^{\star},
$$

where $\Phi^{\star}$ is the viscous dissipation function given by

$$
\Phi^{\star}=2\left[\left(\frac{\partial v^{\star}}{\partial y^{\star}}\right)^{2}+\left(\frac{\partial w^{\star}}{\partial z^{\star}}\right)^{2}\right]+\left(\frac{\partial u^{\star}}{\partial y^{\star}}\right)^{2}+\left(\frac{\partial w^{\star}}{\partial y^{\star}}+\frac{\partial v^{\star}}{\partial z^{\star}}\right)^{2}+\left(\frac{\partial u^{\star}}{\partial z^{\star}}\right)^{2}
$$

where $C_{p}$ is the specific heat at constant pressure, and $\mu$ is the viscosity, $\alpha$ is the thermal diffusivity of the fluid. We assume the temperature at the lower and upper plates are $T_{0}$ and $T_{1}\left(T_{1}>T_{0}\right)$, respectively.

The temperature boundary conditions are

$$
\begin{array}{ll}
T^{\star}=T_{0} & \text { at } y^{\star}=0, \\
T^{\star}=T_{1} & \text { at } y^{\star}=d .
\end{array}
$$

Introducing the nondimensional variables

$$
\theta=\frac{T^{\star}-T_{0}}{T_{1}-T_{0}}, \quad \operatorname{Pr}=\frac{\mu}{\alpha}, \quad E_{c}=\frac{U^{2}}{C_{p}\left(T_{1}-T_{0}\right)}
$$

and on using (2.4), (5.1), and (5.2) become

$$
\operatorname{Pr} \omega \frac{\partial \theta}{\partial t}+\operatorname{Re} \operatorname{Pr}\left(v \frac{\partial \theta}{\partial y}+w \frac{\partial \theta}{\partial z}\right)=\frac{\partial^{2} \theta}{\partial y^{2}}+\frac{\partial^{2} \theta}{\partial z^{2}}+\operatorname{Pr} E_{c} \Phi
$$

where

$$
\Phi=2\left[\left(\frac{\partial v}{\partial y}\right)^{2}+\left(\frac{\partial w}{\partial z}\right)^{2}\right]+\left(\frac{\partial u}{\partial y}\right)^{2}+\left(\frac{\partial u}{\partial z}\right)^{2}+\left(\frac{\partial w}{\partial y}+\frac{\partial v}{\partial z}\right)^{2}
$$

The corresponding boundary condition is reduced to

$$
\theta(0)=0 \quad \theta(1)=1
$$

We assume the solution of the temperature equation in the form

$$
\theta=\theta_{0}(y)+\epsilon \theta_{1}(y, z, t)+\epsilon^{2} \theta_{2}(y, z, t)+\cdots .
$$

Substitute (5.8), (3.1) in (5.13) and equate the term independent of $\epsilon$ and the coefficients of $\epsilon$ and neglect the higher order of $\epsilon$ as $\epsilon \ll 1$. Equating the terms independent of $\epsilon$, 
12 Three-dimensional Couette flow with heat transfer

we get

$$
\theta_{0}^{\prime \prime}+S \operatorname{Re} \operatorname{Pr} \theta_{0}^{\prime}=-\operatorname{PrEc} u_{0}^{\prime 2}
$$

The corresponding temperature boundary conditions are

$$
\theta_{0}(0)=0, \quad \theta_{0}(1)=1
$$

Solving (5.9) under the boundary conditions (5.10), we get

$$
\begin{gathered}
\theta_{0}(y)=h_{1}\left(1-e^{-S \operatorname{RePr} y}\right)+h_{2}\left(e^{-2 S \operatorname{Re} y}-1\right) \quad \text { for } \operatorname{Pr} \neq 2.0, \\
\theta_{0}(y)=D_{1}\left(1-e^{-2 S \operatorname{Re} y}\right)+D_{3} y e^{-2 S \operatorname{Re} y} \quad \text { for } \operatorname{Pr}=2.0,
\end{gathered}
$$

where

$$
\begin{array}{cc}
h_{1}=\frac{1+h_{2}\left(1-e^{-2 S \mathrm{Re}}\right)}{1-e^{-S \operatorname{RePr}}}, \quad h_{2}=\frac{-\operatorname{PrEc}}{2\left(1-e^{-S R e}\right)^{2}(2-\operatorname{Pr})} \quad \text { for } \operatorname{Pr} \neq 2.0, \\
D_{1}=\frac{1-D_{3} e^{-2 S R e}}{1-e^{-2 S R e}}, \quad D_{3}=\frac{E_{c} S \operatorname{Re}}{\left(1-e^{-S R e}\right)^{2}} \quad \text { for } \operatorname{Pr}=2.0 .
\end{array}
$$

Equating the coefficient of $\epsilon$ from both sides, we have

$$
\operatorname{Pr} \omega \frac{\partial \theta_{1}}{\partial t}+\operatorname{Re} \operatorname{Pr}\left[v_{1} \frac{\partial \theta_{0}}{\partial y}+v_{0} \frac{\partial \theta_{1}}{\partial y}\right]=\frac{\partial^{2} \theta_{1}}{\partial y^{2}}+\frac{\partial^{2} \theta_{1}}{\partial z^{2}}+2 \operatorname{PrEc} u_{0}^{\prime} u_{1}^{\prime}
$$

The corresponding conditions for $\theta_{1}$ are

$$
\theta_{1}(0)=0, \quad \theta_{1}(1)=0
$$

We assume the solution of the above differential equation (5.13) of the form

$$
\theta_{1}(y, z, t)=\theta_{11}(y) e^{i(\pi z-t)}
$$

Substituting (5.15) in (5.13) and on using (3.10), we get

$$
\theta_{11}^{\prime \prime}+S \operatorname{Re} \operatorname{Pr} \theta_{11}^{\prime}-\left(\pi^{2}-i \operatorname{Pr} \omega\right) \theta_{11}=\operatorname{Re} \operatorname{Pr} \theta_{0}^{\prime} v_{11}-2 \operatorname{Pr} \operatorname{Ec} u_{0}^{\prime} u_{11}^{\prime}
$$

The corresponding boundary conditions are reduced to

$$
\theta_{11}(0)=0, \quad \theta_{11}(1)=0
$$


The solution of (5.16) subject to the boundary conditions (5.17) and on using (3.4), (3.13), (3.14), and (5.11) are

$$
\begin{aligned}
& \theta_{11}=L\left\{e^{-m_{1} y}-e^{-m_{2} y}\right\} \\
& +S^{2} \operatorname{Re}^{2} \operatorname{Pr}^{2} h_{1}\left[\frac{A}{\lambda_{1}}\left\{e^{-\left(r_{1}+S \operatorname{RePr}\right) y}-e^{-m_{2} y}\right\}+\frac{B}{\lambda_{2}}\left\{e^{-\left(r_{2}+S \operatorname{Re} \operatorname{Pr}\right) y}-e^{-m_{2} y}\right\}\right. \\
& \left.+\frac{C}{\lambda_{3}}\left\{e^{(\pi-S \operatorname{RePr}) y}-e^{-m_{2} y}\right\}+\frac{D}{\lambda_{4}}\left\{e^{-(\pi+S \operatorname{Re} \operatorname{Pr}) y}-e^{-m_{2} y}\right\}\right] \\
& -2 S \operatorname{Re} \operatorname{Pr}\left[\left\{A h_{2} \operatorname{Re}-\frac{\mathrm{Ec} C_{1}\left(r_{1}+S \operatorname{Re}\right)}{\left(1-e^{-S \operatorname{Re}}\right)}\right\} \frac{1}{\lambda_{5}}\left\{e^{-\left(r_{1}+2 S \operatorname{Re}\right) y}-e^{-m_{2} y}\right\}\right. \\
& +\left\{B h_{2} \operatorname{Re}-\frac{\mathrm{Ec} C_{2}\left(r_{2}+S \operatorname{Re}\right)}{\left(1-e^{-S \operatorname{Re}}\right)}\right\} \frac{1}{\lambda_{6}}\left\{e^{-\left(r_{2}+2 S \operatorname{Re}\right) y}-e^{-m_{2} y}\right\} \\
& +\left\{C h_{2} \operatorname{Re}+\frac{\operatorname{Ec} C_{3}(\pi-S \operatorname{Re})}{\left(1-e^{-S \operatorname{Re}}\right)}\right\} \frac{1}{\lambda_{7}}\left\{e^{(\pi-2 S \operatorname{Re}) y}-e^{-m_{2} y}\right\} \\
& \left.+\left\{D h_{2} \operatorname{Re}-\frac{E c C_{4}(\pi+S \operatorname{Re})}{\left(1-e^{-S \operatorname{Re}}\right)}\right\} \frac{1}{\lambda_{8}}\left\{e^{-(\pi+2 S \operatorname{Re}) y}-e^{-m_{2} y}\right\}\right] \\
& +\frac{2 \operatorname{PrEc} S \operatorname{Re}}{\left(1-e^{-S \operatorname{Re}}\right)}\left[\frac{A_{3} r_{1}}{\lambda_{9}}\left\{e^{-\left(r_{1}+S \operatorname{Re}\right) y}-e^{-m_{2} y}\right\}\right. \\
& \left.+\frac{A_{4} r_{2}}{\lambda_{10}}\left\{e^{-\left(r_{2}+S R e\right) y}-e^{-m_{2} y}\right\}\right] \text { for } \operatorname{Pr} \neq 2.0,
\end{aligned}
$$

and for $\operatorname{Pr}=2.0$,

$$
\begin{aligned}
& \theta_{11}(y)=L_{1} e^{-m_{3} y}+M_{1} e^{-m_{4} y}+\left[2 \operatorname{Re} A D_{4}+\frac{C_{1}\left(r_{1}+S \operatorname{Re}\right)}{\beta}\right] \frac{1}{\mu_{1}} e^{-\left(r_{1}+2 S R e\right) y} \\
& +\left[2 \operatorname{Re} B D_{4}+\frac{C_{2}\left(r_{2}+S \operatorname{Re}\right)}{\beta}\right] \frac{1}{\mu_{2}} e^{-\left(r_{2}+2 S \operatorname{Re}\right) y}+\left[2 \operatorname{Re} C D_{4}-\frac{C_{3}(\pi-S \operatorname{Re})}{\beta}\right] \frac{1}{\mu_{3}} \\
& \times e^{(\pi-2 S \operatorname{Re}) y}+\left[2 \operatorname{Re} D D_{4}+\frac{C_{4}(\pi+S \operatorname{Re})}{\beta}\right] \frac{1}{\mu_{4}} e^{-(\pi+2 S \operatorname{Re}) y} \\
& +\frac{1}{\beta}\left[\frac{A_{3} r_{1}}{\mu_{5}} e^{-\left(r_{1}+S R e\right) y}+\frac{A_{4} r_{2}}{\mu_{6}} e^{-\left(r_{2}+S R e\right) y}\right] \\
& -4 S \operatorname{Re}^{2} D_{3}\left[\frac{A}{\mu_{1}}\left\{y+\frac{2\left(r_{1}+S \mathrm{Re}\right)}{\mu_{1}}\right\}\right. \\
& \times e^{-\left(r_{1}+2 S \mathrm{Re}\right) y}+\frac{B}{\mu_{2}}\left\{y+\frac{2\left(r_{2}+S \mathrm{Re}\right)}{\mu_{2}}\right\} e^{-\left(r_{2}+2 S \mathrm{Re}\right) y}+\frac{C}{\mu_{3}} \\
& \left.\times\left\{y-\frac{2(\pi-S \mathrm{Re})}{\mu_{3}}\right\} e^{(\pi-2 S \mathrm{Re}) y}+\frac{D}{\mu_{4}}\left\{y+\frac{2(\pi+S \mathrm{Re})}{\mu_{4}}\right\} e^{-(\pi+2 S \mathrm{Re}) y}\right] \text {, }
\end{aligned}
$$


14 Three-dimensional Couette flow with heat transfer

where

$$
\begin{aligned}
& m_{1,2}=\frac{S \operatorname{Re} \operatorname{Pr}}{2} \pm \frac{1}{2}\left\{S^{2} \operatorname{Re}^{2} \operatorname{Pr}^{2}+4\left(\pi^{2}-i \operatorname{Pr} \omega\right)\right\}^{1 / 2}, \\
& \lambda_{1,2}=S \operatorname{Re} r_{1,2}(\operatorname{Pr}+1.0)+i \omega(\operatorname{Pr}-1.0), \\
& \lambda_{3,4}=\operatorname{Pr}(i \omega \mp S \pi \operatorname{Re}), \\
& \lambda_{5,6}=S \operatorname{Re} r_{1,2}(5-\operatorname{Pr})+2 S^{2} \operatorname{Re}^{2}(2-\operatorname{Pr})+i \omega(\operatorname{Pr}-1.0), \\
& \lambda_{7,8}= \pm \pi S \operatorname{Re}(\operatorname{Pr}-4.0)+2 S^{2} \operatorname{Re}^{2}(2-\operatorname{Pr})+i \operatorname{Pr} \omega, \\
& \lambda_{9,10}=S \operatorname{Re} r_{1,2}(2-\operatorname{Pr})+(1-\operatorname{Pr})\left(S^{2} \operatorname{Pr}^{2}-i \omega\right), \\
& L=\frac{1}{\left(e^{-m_{2}}-e^{-m_{1}}\right)} \\
& \times\left\{S ^ { 2 } \operatorname { R e } ^ { 2 } \operatorname { P r } ^ { 2 } h _ { 1 } \left[\frac{A}{\lambda_{1}}\left\{e^{-\left(r_{1}+S \operatorname{RePr}\right)}-e^{-m_{2}}\right\}\right.\right. \\
& +\frac{B}{\lambda_{2}}\left\{e^{-\left(r_{2}+S \operatorname{RePr}\right)}-e^{-m_{2}}\right\}+\frac{C}{\lambda_{3}}\left\{e^{(\pi-S \operatorname{RePr})}-e^{-m_{2}}\right\} \\
& \left.+\frac{D}{\lambda_{4}}\left\{e^{-(\pi+S \operatorname{RePr})}-e^{-m_{2}}\right\}\right] \\
& -2 S \operatorname{Re} \operatorname{Pr}\left[\left\{A h_{2} \operatorname{Re}-\frac{\mathrm{Ec} C_{1}\left(r_{1}+S \operatorname{Re}\right)}{\left(1-e^{-S \operatorname{Re}}\right)}\right\} \frac{1}{\lambda_{5}}\left\{e^{-\left(r_{1}+2 S \mathrm{Re}\right)}-e^{-m_{2}}\right\}\right. \\
& +\left\{B h_{2} \operatorname{Re}-\frac{\mathrm{Ec} C_{2}\left(r_{2}+S \mathrm{Re}\right)}{\left(1-e^{-S \mathrm{Re}}\right)}\right\} \frac{1}{\lambda_{6}}\left\{e^{-\left(r_{2}+2 S \mathrm{Re}\right)}-e^{-m_{2}}\right\} \\
& +\left\{C h_{2} \operatorname{Re}+\frac{\mathrm{Ec} C_{3}(\pi-S \mathrm{Re})}{\left(1-e^{-S \mathrm{Re}}\right)}\right\} \frac{1}{\lambda_{7}}\left\{e^{(\pi-2 S \mathrm{Re})}-e^{-m_{2}}\right\} \\
& \left.+\left\{D h_{2} \operatorname{Re}-\frac{\operatorname{Ec} C_{4}(\pi+S \operatorname{Re})}{\left(1-e^{-S R e}\right)}\right\} \frac{1}{\lambda_{8}}\left\{e^{-(\pi+2 S R e)}-e^{-m_{2}}\right\}\right] \\
& \left.+\frac{2 \operatorname{PrEc} S \operatorname{Re}}{\left(1-e^{-S \operatorname{Re}}\right)}\left[\frac{A_{3} r_{1}}{\lambda_{9}}\left\{e^{-\left(r_{1}+S \operatorname{Re}\right)}-e^{-m_{2}}\right\}+\frac{A_{4} r_{2}}{\lambda_{10}}\left\{e^{-\left(r_{2}+S R e\right)}-e^{-m_{2}}\right\}\right]\right\} \quad \text { for } \operatorname{Pr} \neq 2.0 \text {, }
\end{aligned}
$$

and for $\operatorname{Pr}=2.0$,

$$
\begin{gathered}
m_{3,4}=S \operatorname{Re} \pm\left\{S^{2} \operatorname{Re}^{2}+\left(\pi^{2}-2 i \omega\right)\right\}^{1 / 2}, \quad \mu_{1,2}=3 S \operatorname{Re} r_{1,2}+i \omega \\
\mu_{3,4}=2 i \omega \mp 2 \pi S \operatorname{Re}, \quad \mu_{5,6}=S \operatorname{Re}\left(r_{1,2}-S \operatorname{Re}\right)+i \omega, \quad \beta=\frac{\left(1-e^{-S \operatorname{Re}}\right)}{4 \operatorname{Ec} S \operatorname{Re}},
\end{gathered}
$$

$L_{1}$ and $M_{1}$ are not given here to save space. 


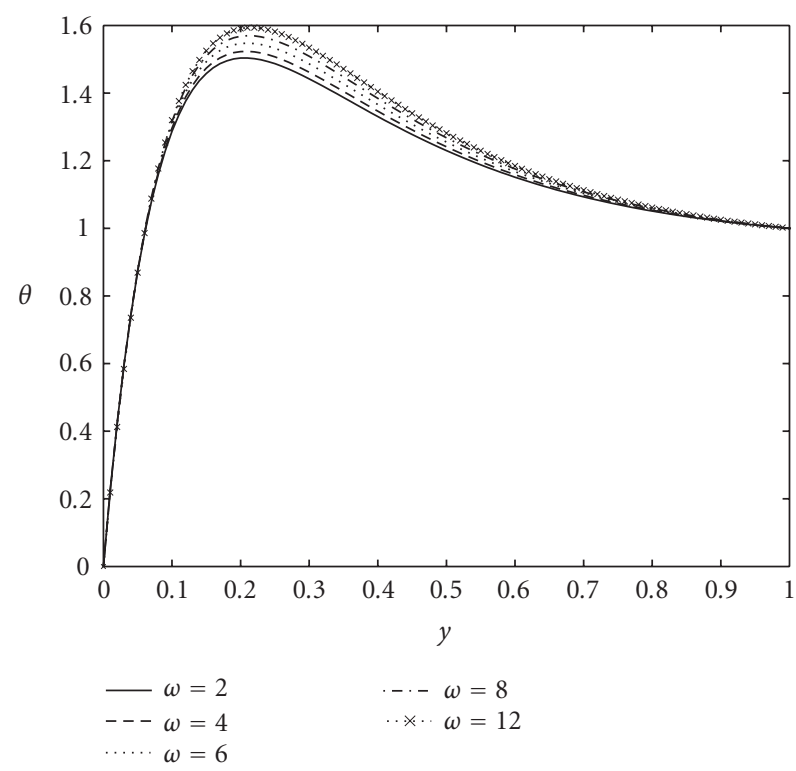

Figure 5.1. Temperature profile for $\operatorname{Re}=5.0, \operatorname{Pr}=0.72, S=1.0, \mathrm{Ec}=10.0, z=0.5, t=0.2, \epsilon=0.2$.

The temperature profile has been plotted against $y$ for different values of frequency parameter $\omega$ and Reynolds number $\operatorname{Re}$ for $\operatorname{Pr}=0.72, \mathrm{Ec}=10.0, S=1.0, z=0.5, t=0.2$ in Figures 5.1 and 5.2. It is observed that the temperature increases with the increase in frequency parameter $\omega$. On the other hand, it increases near the stationary plate and decreases away from the stationary plate with the increase in Reynolds number Re.

One of the most important characteristics of the problem is the rate of heat transfer at the plate. The rate of heat transfer from the plate $y=0$ to the fluid may be calculated using the formula $q_{w}=-(\partial T / \partial y)_{y=0}$ and can be put in nondimensional form in terms of Nusselt number as

$$
\begin{aligned}
N u & =-\frac{q_{w} d}{k\left(T_{1}-T_{0}\right)}=-\left(\frac{d \theta}{d y}\right)_{y=0}=-\left(\frac{d \theta_{0}}{d y}\right)_{y=0}-\epsilon\left(\frac{d \theta_{1}}{d y}\right)_{y=0} \\
& =-\left(\frac{d \theta_{0}}{d y}\right)_{y=0}-\epsilon\left(\frac{d \theta_{11}}{d y}\right)_{y=0} e^{i(\pi z-t)} \\
& =-\theta_{0}^{\prime}-\epsilon \operatorname{Re}_{3} \cos \left(\pi z-t+\phi_{3}\right) \quad \text { for } \operatorname{Pr} \neq 2.0,
\end{aligned}
$$

where

$$
\operatorname{Re}_{3}=\left\{X_{3}^{2}+Y_{3}^{2}\right\}^{1 / 2}, \quad \tan \phi_{3}=\frac{Y_{3}}{X_{3}}
$$




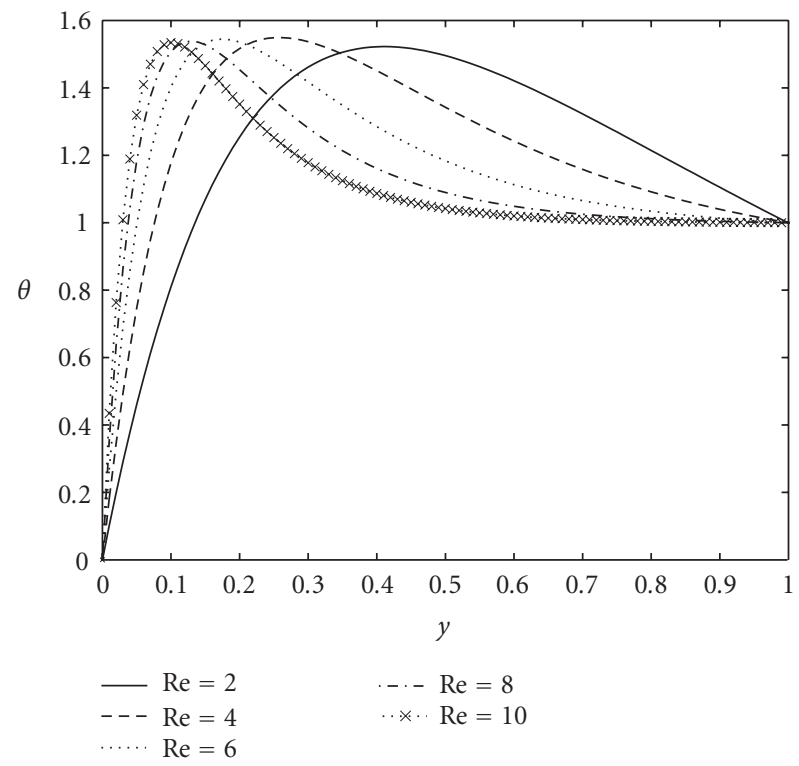

Figure 5.2. Temperature profile for $\operatorname{Pr}=0.72, \omega=6.0, \mathrm{Ec}=10.0, z=0.5, t=0.2, \epsilon=0.2$.

and for $\operatorname{Pr}=2.0$,

$$
\begin{aligned}
N u & =-\frac{q_{w} d}{k\left(T_{1}-T_{0}\right)}=-\left(\frac{d \theta}{d y}\right)_{y=0}=-\left(\frac{d \theta_{0}}{d y}\right)_{y=0}-\epsilon\left(\frac{d \theta_{1}}{d y}\right)_{y=0} \\
& =-\left(\frac{d \theta_{0}}{d y}\right)_{y=0}-\epsilon\left(\frac{d \theta_{11}}{d y}\right)_{y=0} e^{i(\pi z-t)} \\
& =-\theta_{0}^{\prime}-\epsilon \operatorname{Re}_{4} \cos \left(\pi z-t+\phi_{4}\right),
\end{aligned}
$$

where

$$
\operatorname{Re}_{4}=\left\{X_{4}^{2}+Y_{4}^{2}\right\}^{1 / 2}, \quad \tan \phi_{4}=\frac{Y_{4}}{X_{4}}
$$

The rate of heat transfer due to the unsteady part of the temperature is shown in terms of Nusselt number. The amplitude $\mathrm{Re}_{3}$ and the phase shift $\tan \phi_{3}$ in Nusselt number are shown in Figures 5.3 and 5.4 against $\operatorname{Re}$ for $\operatorname{Pr}=0.72, S=1.0$, and $\mathrm{Ec}=10.0$ for different values of $\omega$. It is found that $\operatorname{Re}_{3}$ decreases with the increase in $\omega$. This means that for higher frequencies, rate of heat transfer decreases. Figure 5.1 shows the variation of the phase shift of Nusselt number, $\tan \phi_{3}$ with Re for different values of $\omega$. It is found that for 


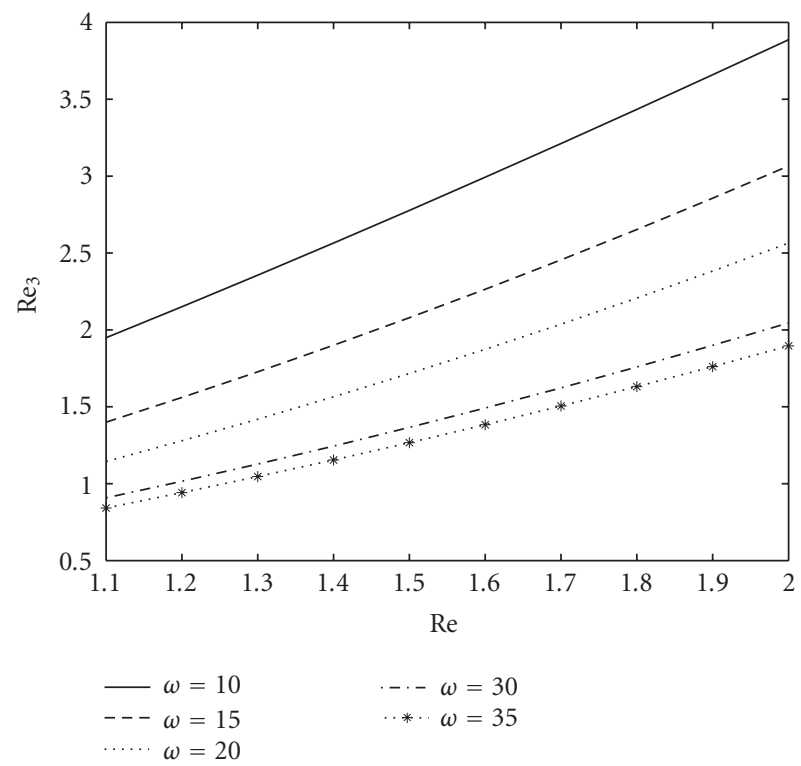

Figure 5.3. The amplitude $\operatorname{Re}_{3}$ of Nusselt number for $\operatorname{Pr}=0.72, S=1.0, \mathrm{Ec}=10$.

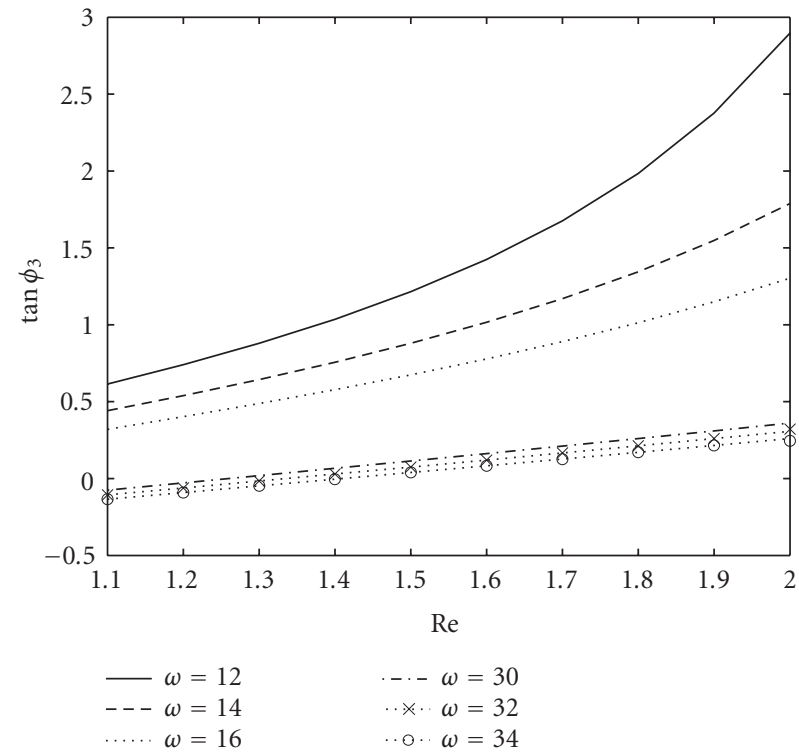

Figure 5.4. Tangent of phase shift of Nusselt number $\tan \phi_{3}$ for $S=1.0, \operatorname{Pr}=0.72, \mathrm{Ec}=10$.

small frequencies there is a phase lead but for large frequencies there is a phase lag. The tangent of the phase shift $\tan \phi_{3}$ increases with the increase in Re. 
18 Three-dimensional Couette flow with heat transfer

\section{Acknowledgment}

M. Guria wishes to thank the University Grants Commission for financial provision for this research.

\section{References}

[1] E. R. G. Eckert, Heat and Mass Transfer, McGraw-Hill, New York, 1958.

[2] K. Gersten and J. F. Gross, Flow and heat transfer along a plane wall with periodic suction, Zeitschrift für Angewandte Mathematik und Physik 25 (1974), 399-408.

[3] H. Schlichting, Boundary Layer Theory, McGraw-Hill, New York, 1979.

[4] K. D. Singh, Three-dimensional viscous flow and heat transfer along a porous plate, Zeitschrift für Angewandte Mathematik und Mechanik 73 (1993), no. 1, 58-61.

[5] _ Three dimensional couette flow with transpiration cooling, Zeitschrift für Angewandte Mathematik und Physik 50 (1999), no. 4, 661-668.

[6] P. Singh, V. P. Sharma, and U. N. Misra, Three dimensional fluctuating flow and heat transfer along a plate with suction, International Journal of Heat and Mass Transfer 21 (1978), no. 8, 1117-1123.

[7] - Three dimensional free convection flow and heat transfer along a porous vertical plate, Applied Scientific Research 34 (1978), no. 1, 105-115.

M. Guria: Department of Applied Mathematics, Vidyasagar University,

Midnapore 721 102, India

E-mail address: mrinmoy9832@yahoo.com

R. N. Jana: Department of Applied Mathematics, Vidyasagar University,

Midnapore 721 102, India

E-mail address: jana261171@yahoo.co.in 


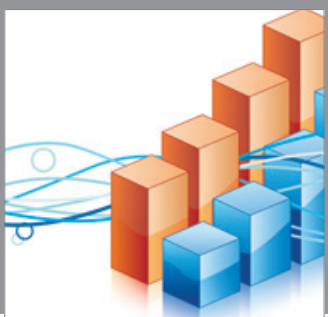

Advances in

Operations Research

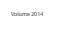

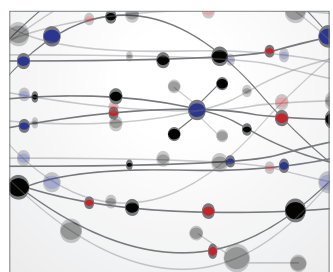

\section{The Scientific} World Journal
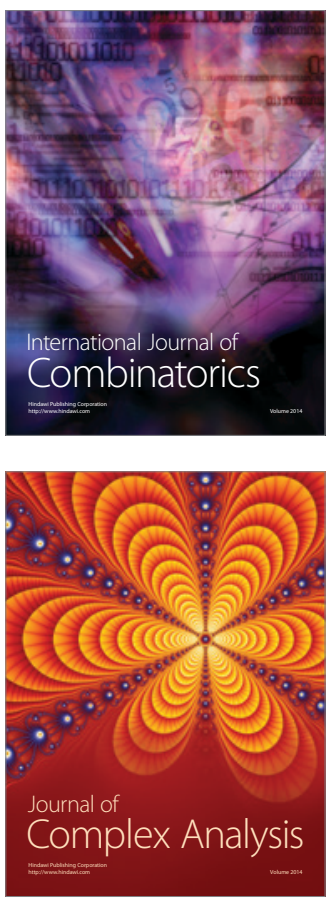

International Journal of

Mathematics and

Mathematical

Sciences
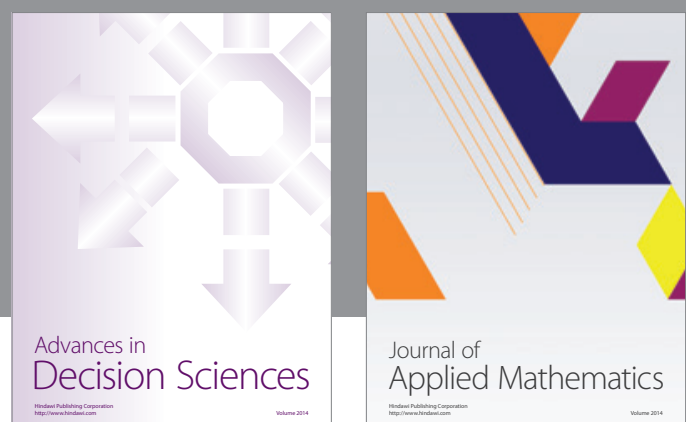

Journal of

Applied Mathematics
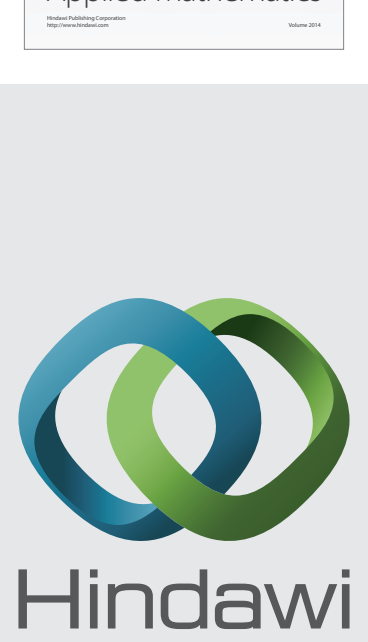

Submit your manuscripts at http://www.hindawi.com
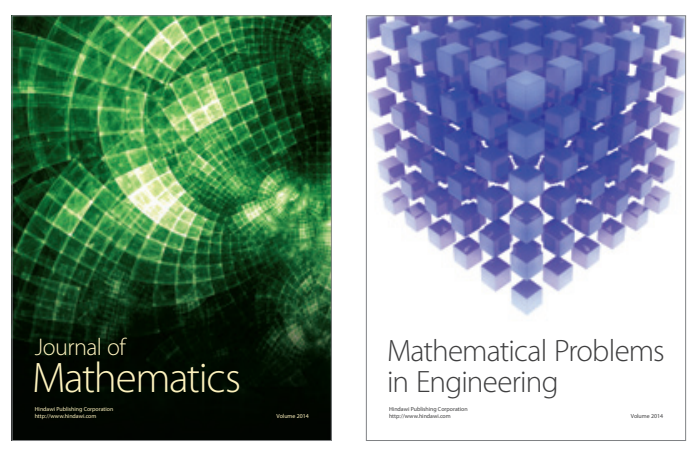

Mathematical Problems in Engineering
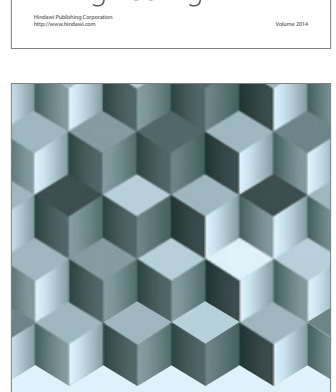

Journal of

Function Spaces
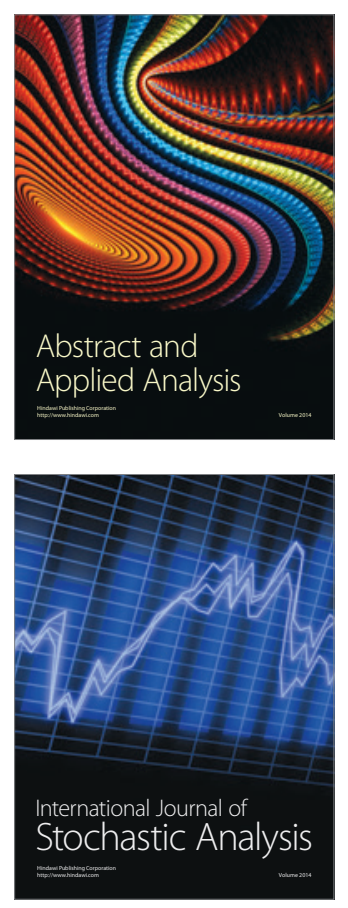

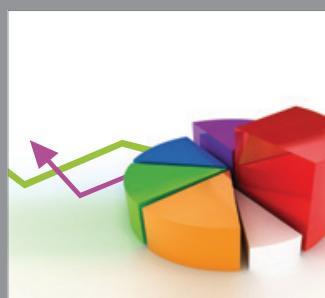

ournal of

Probability and Statistics

Promensencen
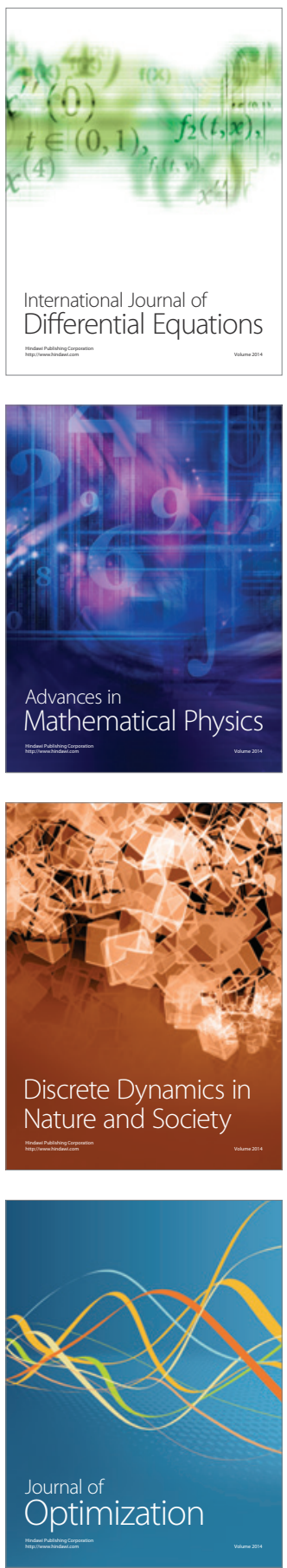